\title{
Performance Analysis of Cognitive Radio based WRAN over Rayleigh Fading Channel with Alamouti-STBC 2X1, 2X2\&2X4 Multiplexing
}

\author{
Rahul Koshti \\ Assistant Professor \\ Narsee Monjee Institute of Management Studies \\ MPSTME, Shirpur (MH.)
}

\begin{abstract}
Bit error rate (BER) is the fundamental parameter to access the quality of any wireless communication. The good quality (low BER) of transmission over less SNR value is always a topic of research. However the BER can be reduced by increasing the SNR, the high value of SNR for wireless communication is limited by available transmitted power \& large distance. It is observed that different modulation techniques struggles neck to neck for getting low BER, at lesser value of SNR but still with the slight change in BER the quality of transmission changes many folds. In this work we are evaluating the BER performance of cognitive radio based IEEE 802.22 WRAN with ALAMOUTI-STBC multiplexing over Rayleigh fading channel using BPSK \& QPSK modulation in MATLAB environment.
\end{abstract}

\section{Keywords}

WRAN, cognitive radio, BER, Alamouti-STBC

\section{INTRODUCTION}

The wireless Technology uses three concepts 1 . Adaptive radio, 2.cognitive radio, 3.intelligent radio. For IEEE 802.22 wireless regional area network (WRAN) standard cognitive radio concept is implemented. Cognitive radio is a software defined radio technology that automatically selects the communication parameters depending upon the available spectrum. It is the communication system in which transmitter and receiver changes their operating parameters efficiently and dynamically [1] without interfering the licensed users. Since this technology detects holes (free spectrum) from the allocated one, makes the spectrum utilization effective. The spectrum utilization is shown in fig 1 .

As shown in fig 1 from the total allocated spectrum some of the spectrum is free, in order to identify the licensed user and detect the holes (unused spectrum), the system has to sense the spectrum. Signal processing algorithms are used for spectrum sensing. These algorithms are match filtering, energy detection and cyclostationary feature detection [2]. However in communication system normally the match filtering is the effective way of signal detection at the same time it offers the drawback of requirement of prior knowledge of licensed user signal. Also in the energy detection algorithm detection is subjected to changing SNR

$$
x=\left[\begin{array}{ll}
x_{1} & x_{2} \\
x_{2}^{*} & x_{1}^{*}
\end{array}\right]
$$

\author{
Mayank Kothari \\ Assistant Professor \\ Narsee Monjee Institute of Management Studies, \\ MPSTME, Shirpur (MH.)
}

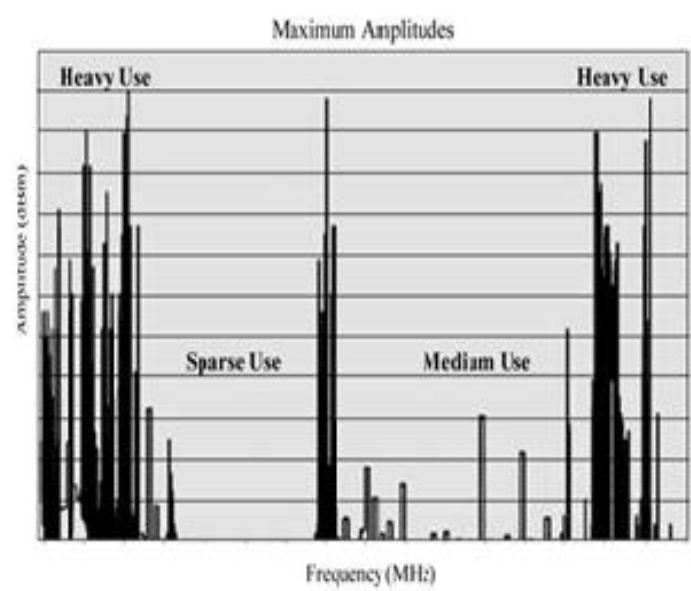

Fig 1: frequency spectrum utilization

Further it cannot differentiate interference from the user signal therefore the energy detection is not effective for spectrum sensing. As an alternate to these two methods cyclostationary detection algorithm is best suited for signal detection for spectrum sensing. The architecture of cyclostationary energy detection is shown in fig. 2.

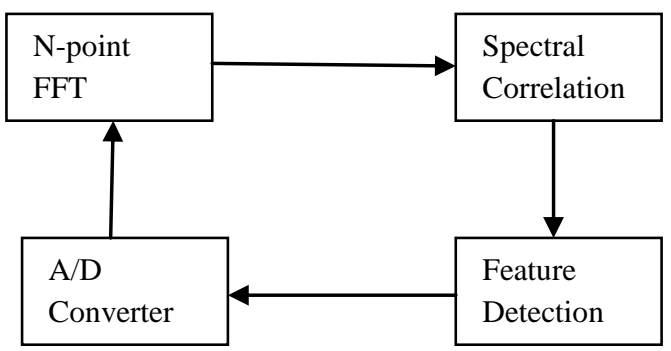

Fig.2: Architecture of Cyclostationary detection

Here by varying the size of FFT, spectrum sensing system for different frequencies can be designed

\section{ALAMOUTI-STBC MULTIPLEXING}

In wireless communication system in order to improve the reliability of data transfer multiple copies of data is transmitted across the number of antenna. This technique of data transfer is deployed using space time block code (STBC) Alamouti published this scheme for data transmission [4].

Alamouti's scheme was the first STBC [3]. The Alamouti If the duration of each symbol is $\mathrm{T}$ then at time $\mathrm{t}+\mathrm{T}$ symbol $\mathrm{x} \_2 *$ and $\mathrm{x} \_1 *$ are transmitted. Where $*$ represents complex conjugate. In this scheme the minimum transmitting antenna 
should be 2 and $\mathrm{N}$ receiving antenna. The decoding scheme for a 2X1 system (Two transmitter and one receiver) is illustrated below [4] STBC technique uses two transmitting antenna and $\mathrm{N}$ number of receiving antenna and can support a diversity order of $2 \mathrm{~N}$ with code rate 1 (full rate) [4]. The encoding scheme of Alamouti is given in [5]. In this scheme different time instant and transmitted symbol are represented in matrix form. Row of the matrix represents the time instant while column represents transmitted symbol through different antenna. As per this scheme the transmission of symbol S1 and $\mathrm{S} 2$ at time $\mathrm{t}$ from antenna 1 and antenna 2 respectively is shown by the following matrix

$$
\begin{aligned}
& r_{1}^{(1)}=r_{1}(t)=h_{1,1} x_{1}+h_{1,2} x_{2}+n_{1}^{(1)} \\
& r_{1}^{(2)}=r_{1}(t+T)=-h_{1,1} x_{2}^{*}+h_{1,2} x_{1}^{*}+n_{1}^{(2)}
\end{aligned}
$$

In the above equations $r_{1}$ is the received signal at antenna 1. $h_{i, j}$ is the channel transfer function from the $j^{\text {th }}$ transmitting antenna and $i^{\text {th }}$ receiving antenna. $n_{1}$ Represents noise at antenna 1 and it is a complex random variable.

The received signals are combined together as illustrated below before they are sent to the STBC decoder [4].

$$
\begin{aligned}
& x_{1}^{\prime}=h_{1,1}^{*} r_{1}^{(1)}+h_{1,2} r_{1}^{*(2)} \\
& x_{2}^{\prime}=h_{1,2}^{*} r_{1}^{(1)}+h_{1,1} r_{1}^{*(2)}
\end{aligned}
$$

Once the received signals are combined they are sent to the maximum likelihood decoder for the estimation of transmitted signal $x_{1}$ and $x_{2}$.

In the same way the STBC-multiplexing scheme for two transmitters and two receivers $(2 \mathrm{X} 2)$ is shown below

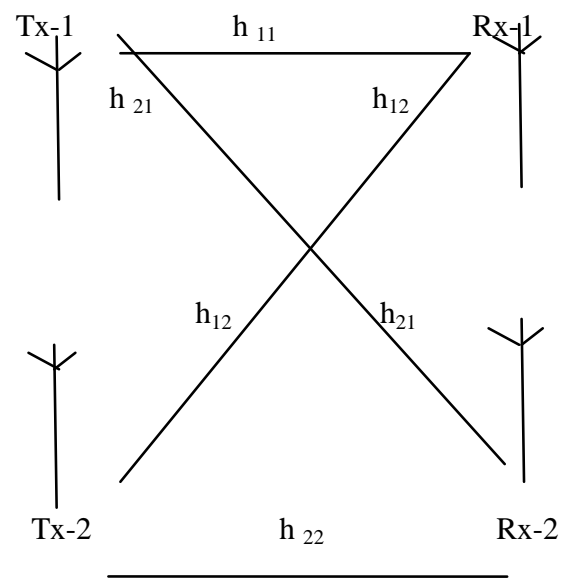

Fig.3: 2X2 STBC multiplexing

Here if we assume that the channel parameters remains constant, the encoding is done in the same way as in previous case. Here the received symbols are [4].

$$
\begin{aligned}
& r_{1}^{(1)}=h_{1,1} x_{1}+h_{1,2} x_{2}+n_{1}^{(1)} \\
& r_{1}^{(2)}=-h_{1,1} x_{2}^{*}+h_{1,2} x_{1}^{*}+n_{1}^{2} \\
& r_{2}^{(1)}=h_{2,1} x_{1}+h_{2,2} x_{2}+n_{2}^{(1)} \\
& r_{2}^{(2)}=-h_{2,1} x_{2}^{*}+h_{2,2} x_{1}^{*}+n_{2}^{(2)}
\end{aligned}
$$

The combined signal before they are sent to the decoder are-

$$
\begin{aligned}
& x_{1}^{\prime}=h_{1,1}^{*} r_{1}^{(1)}+h_{1,2} r_{1}^{*(2)}+h_{2,1}^{*} r_{2}^{(1)}+h_{2,2} r_{2}^{*(2)} \\
& x_{2}^{\prime}=h_{1,2}^{*} r_{1}^{(1)}+h_{1,1} r_{1}^{*(2)}+h_{2,2}^{*} r_{2}^{(1)}+h_{1,1} r_{2}^{*(2)}
\end{aligned}
$$

\section{PROPOSED SIMULATION MODEL OF WRAN}

IEEE 802.22 WRAN is the first cognitive radio technology for efficient utilization of available spectrum. It operates in the rural area provides broadband access to the data networking using vacant TV channels in VHF \& UHF bands. It operates in $54 \mathrm{MHz}$ to $862 \mathrm{MHz}$ band [6]. Its coverage range is $33 \mathrm{Km} .-100 \mathrm{Km}$

The simulation model is divided in transmitter and receiver section. At the transmitter the random data is generated. By randomization binary sequence is converted in to random sequence to avoid long sequence of ' $o$ 's and ' 1 ' s. here XOR operation is performed with 15 bit linear feedback shift register (LFSR). Randomized data is then coded for forward error correction using convolution code or reed Solomon code

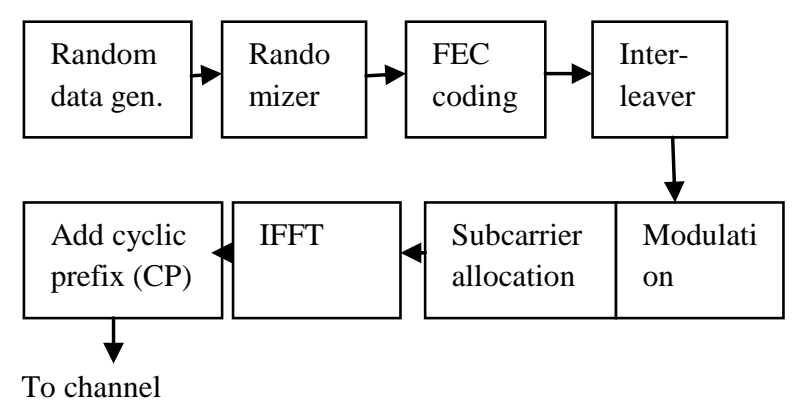

Fig.4: Transmitter of WRAN

Interleaving is Similar to randomization but changes only the position of the bits not the state of the bits. The aim is to achieve desirable bit error distribution after demodulation. The incoming data into the Interleaver is randomized in two permutations. First permutation ensures that adjacent bits are mapped onto nonadjacent subcarriers. The second permutation maps the adjacent coded bits onto less or more significant bits of constellation. Same permutation is done on the receiver side to rearrange the data bits into the correct sequence.

Modulation is a Fundamental process of a digital communication system. It involves mapping of data bits on to the subcarrier. Two modulations scheme namely BPSK, QPSK have been used in this work.

OFDMA subcarrier allocation scheme is used for multiuser OFDM system. Now pilots are inserted within the data subcarriers. Pilot are Used for various estimation and synchronization purposes. IFFT converts the data from frequency domain to time domain data representing OFDM subcarrier. It Generate samples of a waveform with frequency component satisfying orthogonally condition. By the addition of Cyclic Prefix ISI can be prevented. CP copies the last section of a symbol \& paste it to the beginning of symbol. It performs as a Guard interval 


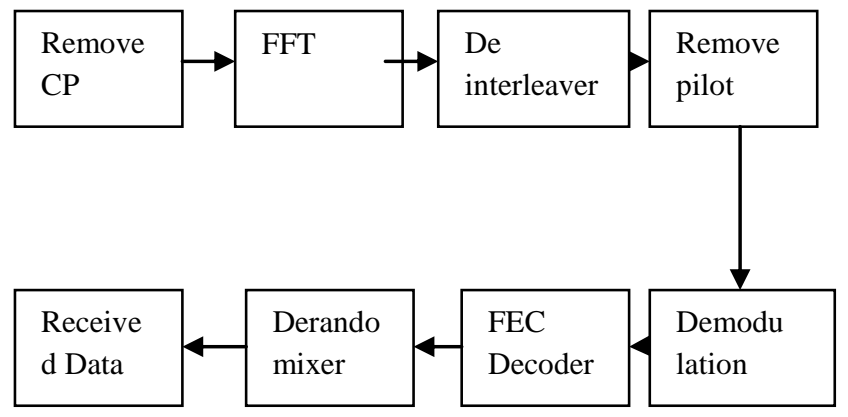

Fig.5: Receiver of WRAN

In this work Rayleigh channel is taken into consideration for the simulation purpose.

At the receiving side, a reverse process (including deinterleaving and decoding) is executed to obtain the original data bits.

\section{SIMULATION DETAILS}

IEEE 802.22 WRAN System is simulated over physical layer using Rayleigh channel in MATLAB 7.6. Following parameters of WRAN are used for Simulation

Table-1 Simulation parameter

\begin{tabular}{|l|l|l|}
\hline S. No. & \multicolumn{1}{|c|}{ Parameter } & Value \\
\hline 1 & Total No. of subcarriers & 2048 \\
\hline 2 & No. of Data Subcarriers & 1440 \\
\hline 3 & Pilot \& guard bits & 608 \\
\hline 4 & Sampling Frequency & $6 \mathrm{Mhz}$ \\
\hline 5 & Code rate & $1 / 2$ \\
\hline 6 & Cyclic prefix & $1 / 4$ \\
\hline 7 & Modulation method & BPSK\& QPSK \\
\hline 8 & Channel & Rayleigh \\
\hline
\end{tabular}

For the simulation random data is generated consisting of 10 Symbols. After converting Serial data into parallel form convolution coding is used as FEC code. Then Modulation is performed. Interleaving is used to convert burst error in to random error. After addition of pilot \& CP bits Alamouti coding is implemented and signal is passed through the Rayleigh channel. Reverse process is performed at the receiver. The Simulation curves are obtained between BER \& SNR which are shown in subsequent graph

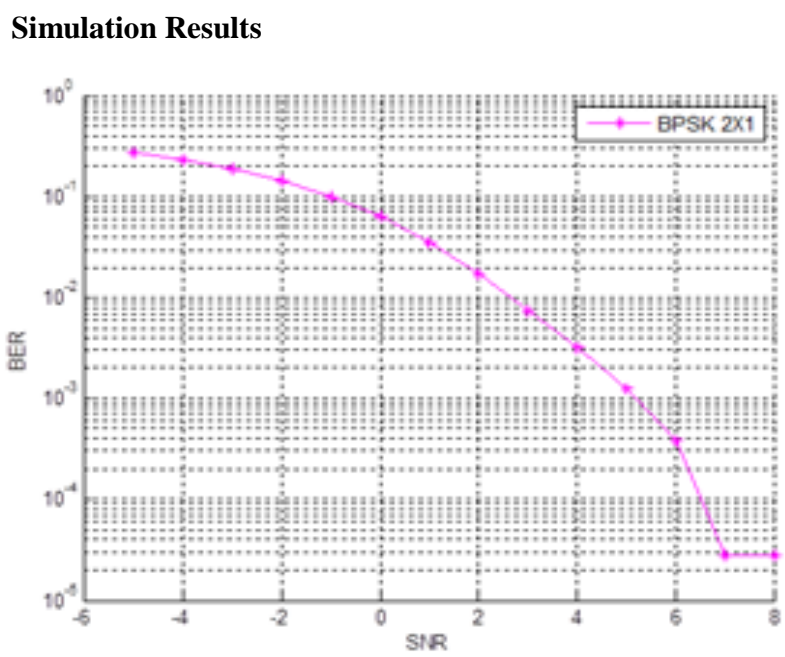

Fig.6: BPSK-2X1 WRAN

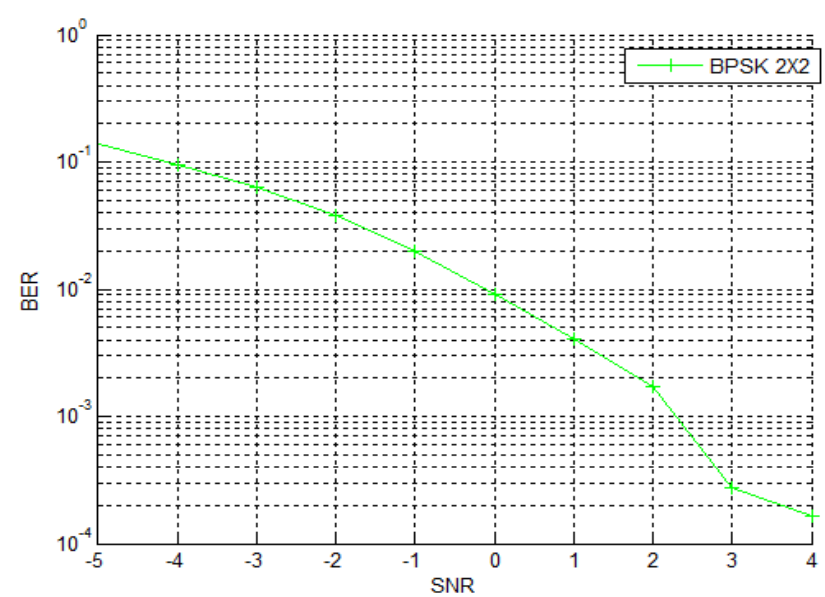

Fig.7: BPSK-2X2 WRAN

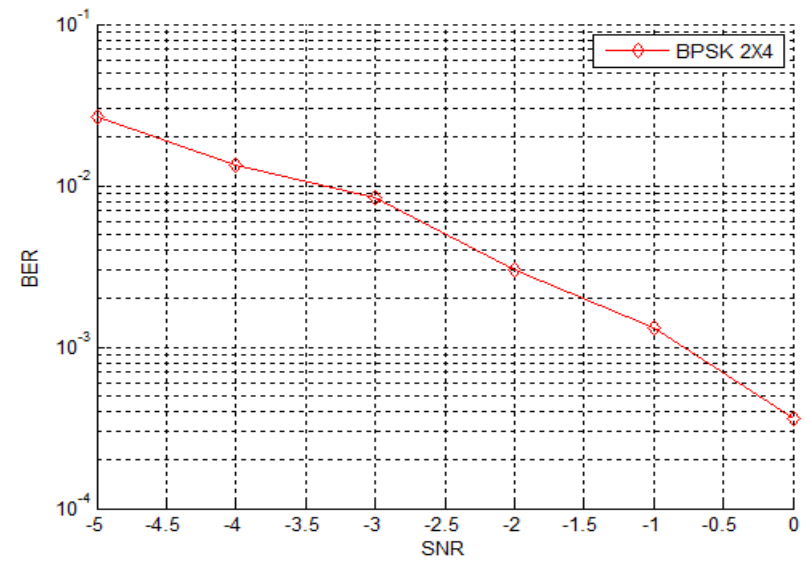

Fig.8: BPSK-2X4 WRAN

As shown in fig 6(2X1 WRAN with BPSK Modulation) for a fixed values of BER i.e. 10-3 the SNR is approximately $5 \mathrm{db}$. While for a $2 \mathrm{X} 2$ system for the same value of BER (10-3) SNR is slightly greater than $2 \mathrm{db}$ shown in fig.7. While for a $2 \mathrm{X} 4$ system for the BER of 10-3, SNR is $-1 \mathrm{db}$ it is shown in fig 8 . All these results are obtained for BPSK modulation. 


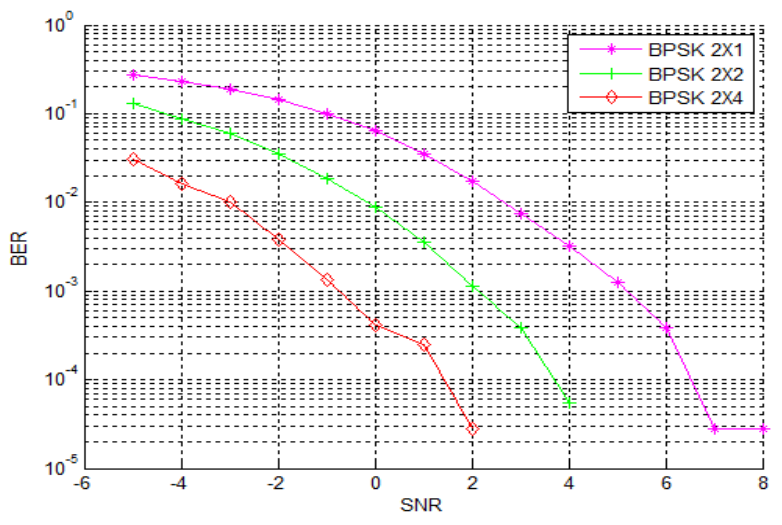

Fig.9: Comparison of 2X1, 2X2, 2X4 BPSK-WRAN

Now with the same parameters the BER V/S SNR curves are obtained with QPSK modulation for 1,2,4 receivers respectively shown in the subsequent graph.

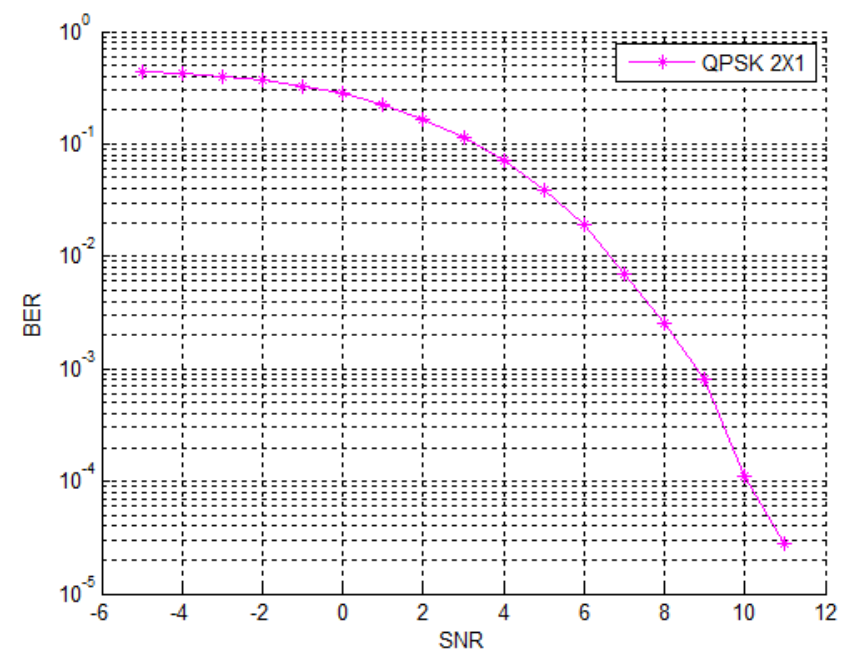

Fig.10: QPSK 2X1 WRAN

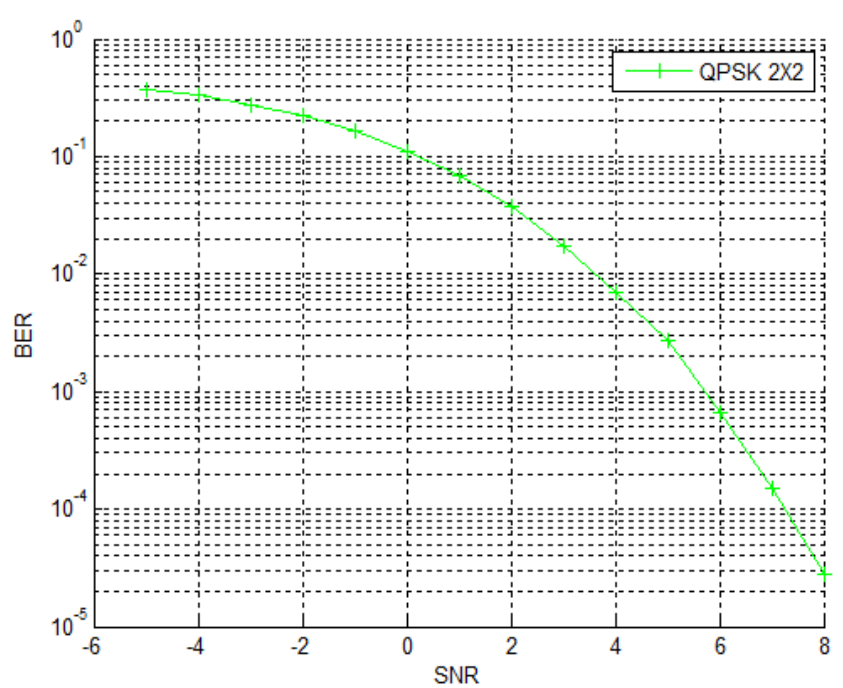

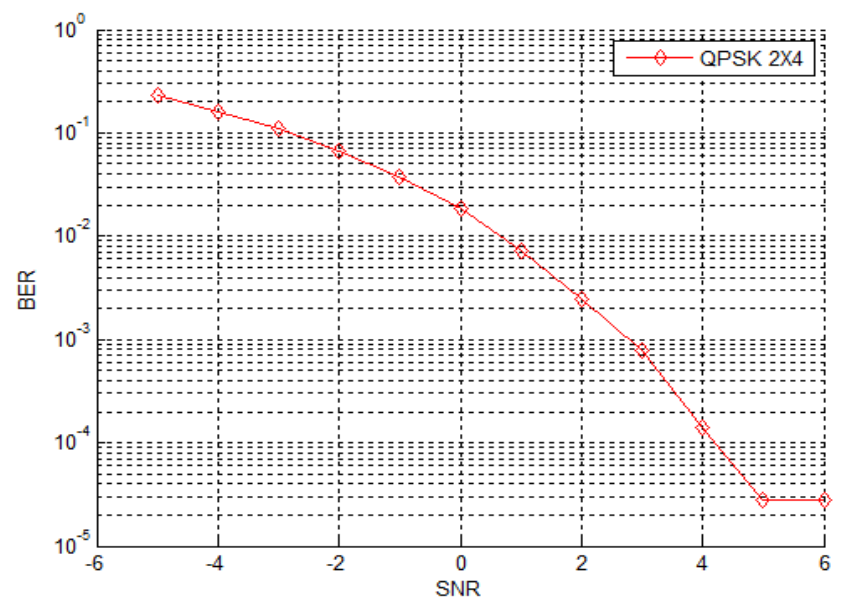

Fig.12: QPSK-2X4 WRAN

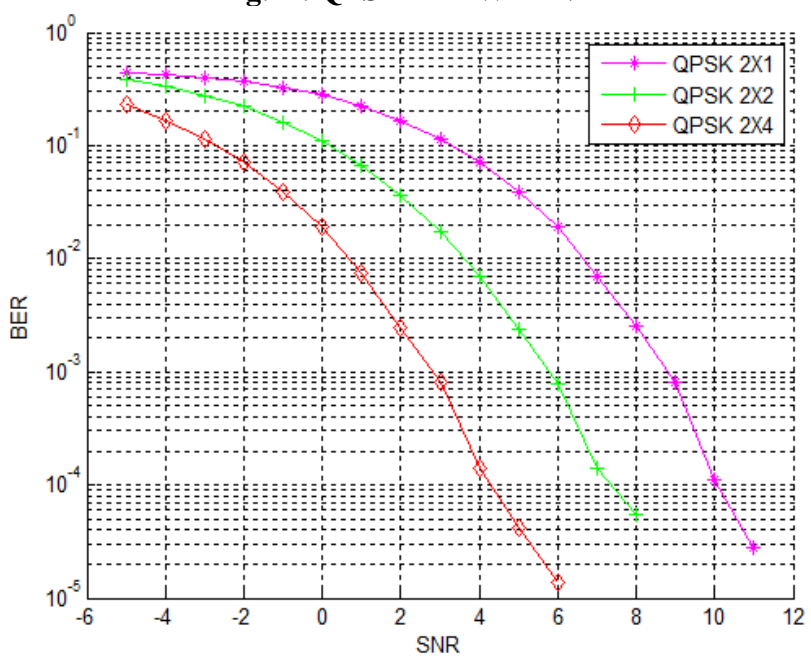

Fig.13: Comparison 2X1, 2X2, 2X4 QPSK-WRAN

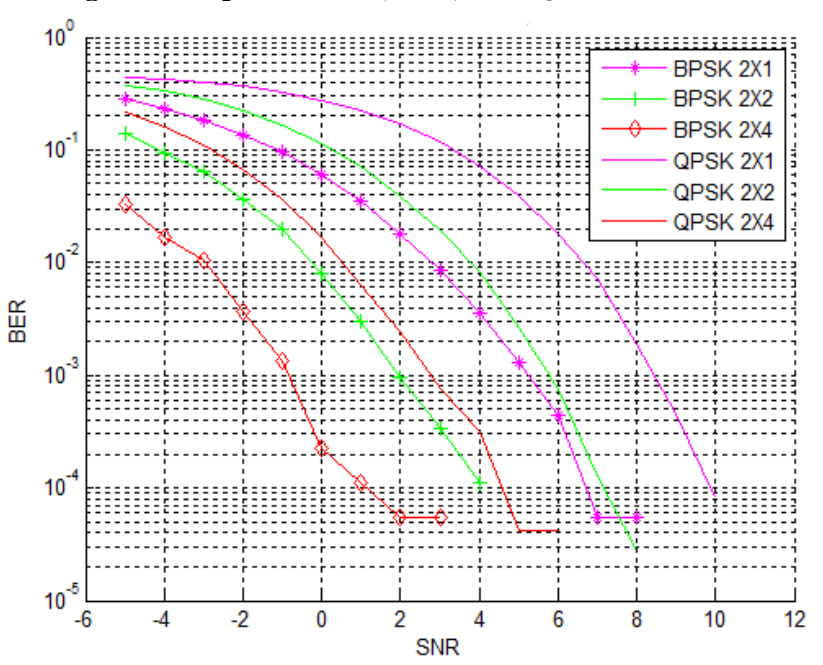

Fig.14: Comparison of BPSK \& QPSK 2X2, 2X2, 2X4 WRAN

Fig.11: QPSK-2X2 WRAN 
For a 2X1 system with QPSK Modulation, for a BER of $10^{-3}$ the SNR is approximately $9 \mathrm{~dB}$ shown in fig 10 . While for a $2 \mathrm{X} 2$ system for the same BER, SNR is greater than $5 \mathrm{~dB}$ it is shown in fig 11 . For a $2 X 4$ system with QPSK modulation the SNR is about $3 \mathrm{~dB}$ for a BER of $10^{-3}$ in comparison with 2X1 system shown in fig 12 .

\section{CONCLUSION}

In this work, IEEE 802.22 WRAN System is simulated over physical layer using Rayleigh channel in MATLAB. The simulation results are obtained between BER vs. SNR. From the graphs it is observed that the performance of WRAN over Rayleigh fading Channel is significantly improved with Alamouti Coding. Since the principle objective of this project is to improve the performance of the system by fixing up the BER for a smaller value of SNR, hence the rapid decay in the simulation curve shows the better performance. Also the system gives better performance with BPSK modulation. So it is concluded that the performance of WRAN over Rayleigh fading channel is better with Alamouti coding and BPSK Modulation.

\section{REFERENCES}

[1] Mitola J. 2000, Cognitive radio-an integrated agent architecture for software defined radio. Dissertation Stockholm

[2] Danijela Cambric, Sridhar Mubaraq Mishra, Robert W. Brodersenet, 2004, Implementation issues in spectrum sensing for cognitive radios.
[3] G. Tsoulos, 2006, MIMO system technology for wireless communications, CRC press.

[4] S. Alamouti, 1998, "A simple transmit diversity technique for wireless communications," IEEE Journal on selected areas in communications, vol. 16, no. 8, pp. 1451-1458, “IEEE P802.11n/D5.0,” May 2008.

[5] Carl R. Stevenson, Gerald Chouinard, Zhongding Lei, Wendong $\mathrm{Hu}$, Stephen J. Shellhammer and Winston Caldwell, January 2009, "IEEE 802.22: The First Cognitive Radio Wireless Regional Area Network Standard" IEEE Communications Magazine.

[6] S.H. Hwang, J.S. Um, M.S. Song, C.J. Kim, H.R. Park, and Y.H. Kim, IEEE Press, May. 2008 "Design and Verification of IEEE 802.22 WRAN Physical Layer (Invited Paper)," Proc. International Conf. Cognitive Radio Oriented Wireless Networks and Communications (CrownCom 08), pp. 1-6

[7] K. Cordeiro, D. Challapali, D.Birru, and S.Shankar, "IEEE 802.22, Nov. 2005 the First Worldwide Wireless Standard based on Cognitive Radios," Proc. IEEE Symp. New Frontiers in Dynamic Spectrum Access Networks (DySPAN 05), pp. 328-337

[8] Upena dalal "Wireless Communication System", Oxford University press 